\title{
Analysis of the Redundant Energy in UW-OFDM
}

\author{
Heidi Steendam, Senior Member, IEEE
}

\begin{abstract}
In this paper, we consider unique-word (UW) OFDM, where the guard interval, filled with known samples, is a part of the inverse FFT output. In order to construct the known samples in the UW-OFDM signal, a number of carriers needs to be reserved as redundant carriers. The information modulated on these redundant carriers depends on the transmitted data. However, the energy transmitted on the redundant carriers strongly depends on the positions of these redundant carriers. In this paper, we consider the distribution of the redundant carriers from [1] that is suboptimal but yields an average redundant energy that is close to the minimum, and analytically evaluate the redundant energy as a function of the system parameters, i.e. the FFT length, the number of redundant carriers and the length of the unique word. Based on our results, a simple rule of thumb can be obtained for the selection of the system parameters, which is helpful in the design of UW-OFDM systems.
\end{abstract}

Index Terms-Multicarrier systems, redundant carriers, energy consumption.

\section{INTRODUCTION}

$\mathbf{I}$ $\mathrm{N}$ multicarrier systems like OFDM, the bandwidth is divided into a large number of narrow subchannels, i.e. the subcarriers; the data is transmitted in parallel over these subcarriers and converted to a time-domain signal using an inverse FFT (IFFT). Because of the narrow bandwidth of these subcarriers, the subchannel can be considered frequencyflat, which implies that multicarrier systems are robust against channel dispersion. Hence, multicarrier transmission is able to achieve a high bandwidth efficiency in dispersive channels and is therefore the basis for various standards [2]-[4]. In order to avoid that sequentially transmitted OFDM blocks overlap in time and cause intersymbol interference, traditionally a guard interval is inserted between the different IFFT blocks. There exist several guard interval techniques like cyclic prefix, zero padding and known symbol padding [5]-[6]. Common to these techniques is the extension of the length of the OFDM symbol in the time domain which reduces the effective throughput of the OFDM system. Recently, a new approach has been introduced in [7], i.e. unique-word (UW) OFDM. In this technique, in contrast with the traditional guard interval techniques, the guard interval is a part of the IFFT block: the last part of an IFFT block in UW-OFDM does not depend on the transmitted data but is a known sequence, the unique word. To be able to construct this unique word, however, we have to sacrifice subcarriers - these subcarriers are called redundant subcarriers.

Paper approved by H. Minn, the Editor for Synchronization and Equalization of the IEEE Communications Society. Manuscript received August 24, 2011; revised January 24, 2012.

The author is with DIGCOM Research Group, TELIN Dept., Ghent University, Sint-Pietersnieuwstraat 41, 9000 Gent, Belgium (e-mail: Heidi.Steendam@telin.ugent.be).

Digital Object Identifier 10.1109/TCOMM.2012.041212.110560
In [8], two different methods were introduced to construct UW-OFDM. In the direct approach, the unique word is directly formed by properly selecting the information modulated on the redundant subcarriers, whereas in the two-step approach, first the information modulated on the redundant carriers is selected such that the part of the time domain signal corresponding to the unique word is set to zero, and the unique word itself is added afterwards. In [8], it is shown that the two-step approach requires less redundant energy than the direct approach. As in the two-step approach first a signal is generated with a block of zeros in the time domain, the UW-OFDM technique can be compared with a Reed-Solomon code [9].

When we compare the throughput efficiency of UW-OFDM with that of CP-OFDM, it can easily be verified that, assuming the same bandwidth and guard interval duration, and a guard interval length equal to $25 \%$ of the FFT length, the throughput efficiency equals $75 \%$ in UW-OFDM and $80 \%$ in CP-OFDM. Hence, the throughput efficiency in UW-OFDM is slightly lower than in CP-OFDM. This implies that in an AWGN channel, CP-OFDM will slightly outperform UW-OFDM: this effect is shown in [8]. However, UW-OFDM will outperform CP-OFDM in frequency selective channels. This has been shown in [8] for the uncoded case and [7] and [10] for the coded case. This can be explained by the redundancy that is present in the frequency domain: translating the UWOFDM system in coding terms, the redundancy will introduce a coding gain as compared to CP-OFDM. The effect of this coding gain will be larger when there are more deep fades in the channel. Note that the effect of deep fades in CPOFDM can be counteracted by using precoding. However, this requires the presence of channel state information (CSI), which is difficult to obtain timely and sufficiently accurate at the transmitter. In UW-OFDM, such CSI is not necessary. The performance gain in UW-OFDM however comes with a price in complexity: whereas in CP-OFDM a simple linear data detector is sufficient, sophisticated detectors that fully exploit the redundancy in the signal are necessary for UW-OFDM. Further, the information in the guard interval in CP-OFDM depends on the data symbols and is thus prior unknown at the receiver, whereas in UW-OFDM, the information in the guard interval does not depend on the data symbols, and is prior known at the receiver. The known symbols in the guard interval can therefore easily be used for parameter estimation. In this sense, UW-OFDM is similar to KSP-OFDM, where it is shown that channel estimation and synchronization is more accurate than in CP-OFDM thanks to the presence of the known samples in the guard interval [11]-[13].

In [7] and [8], the authors considered only the case where the number of redundant carriers equals a power of two. In that case, it was shown through simulations that a (close 
to) uniform distribution ${ }^{1}$ for the redundant carriers over the bandwidth resulted in the minimum average redundant energy. In this paper, we analytically prove that the uniform distribution for this case is indeed optimal. When the number of redundant carriers is no longer a power of two, the situation becomes more complex. In [1], it is shown that the uniform distribution is no longer optimal. Moreover, the energy needed for the redundant carriers strongly depends on the positions of the redundant carriers - this redundant energy increases exponentially when the redundant carrier positions are not carefully selected. So it is of major importance to use an appropriate redundant carrier distribution. We select the distribution that minimizes the average redundant energy. For the general case where $N_{r}$ is not a power of 2, the minimization of the average redundant energy is a computationally hard problem: an exhaustive search must be carried out to find the optimal positions. For small values of $N(N \leq 32)$, the optimal positions were determined through an exhaustive search, but for larger values of $N(N>32)$, this was practically not possible. No simple rule of thumb was found to extend the results for small values of $N$ to larger values of $N$ to obtain the positions for the redundant carriers that minimize the average redundant energy. However, in [1], a distribution for the redundant carriers is proposed where the redundant carrier positions are selected according to a simple analytical expression. For small values of $N$, this distribution yielded for almost all cases an average redundant energy relatively close to the minimum obtained with the exhaustive search. For larger values of $N$, extensive simulations were carried out to find the optimal distribution. The distribution from [1] in this case always gave the lowest average redundant energy. Hence, the distribution from [1] is a good compromise between optimality and complexity.

In this paper, we consider the distribution from [1] and analytically evaluate the effect of the system parameters (i.e. the FFT length, the length of the unique word and the number of redundant carriers) on the average redundant energy. For the special case where the number of redundant carriers equals the unique word length, a closed expression for the average redundant energy is derived. For the general case, where the number of redundant carriers is larger than the unique word length, the average redundant energy can indirectly be calculated. From our analysis, we are able to derive a simple rule of thumb for the selection of the system parameters such that the redundant energy is small. This rule of thumb is useful for the design of UW-OFDM systems.

\section{SySTEM DESCRIPTION}

We use the two-step approach to construct the UW-OFDM signal [8]. The time-domain signal consists of blocks of length $N$ (where $N$ is a power of 2) samples generated at a sample rate $1 / T$. In a block of $N$ time-domain samples, the first

${ }^{1}$ In [7], the authors consider guard bands in the frequency domain where no information can be transmitted. With a uniform distribution, some of the redundant carriers should be placed on a zero carrier belonging to the guard band, which is not allowed. The optimization in [7] takes into account the restriction of the guard bands and results in a close to uniform distribution where some of the redundant carriers are shifted as compared to the uniform distribution. In this paper, however, we do not take into account guard bands.

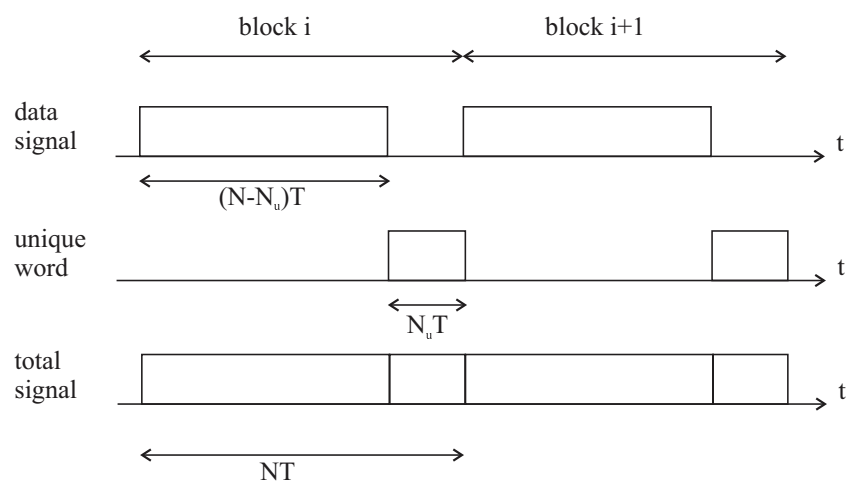

Fig. 1. Time-domain signal of UW-OFDM.

$N-N_{u}$ samples depend on the data and the last $N_{u}$ samples contain the known sequence corresponding to the unique word, as shown in Figure 1. To be able to construct in the first step the block of $N_{u}$ zeroes in the time domain, we need to replace $N_{r}$ data carriers by redundant carriers, where $N_{r} \geq N_{u}$. We define $\mathbf{x}_{d}=\left(x_{d}(1) \ldots x_{d}\left(N-N_{r}\right)\right)^{T}$ as the vector of $N_{d}=N-N_{r}$ data symbols and $\mathbf{x}_{r}=\left(x_{r}(1) \ldots x_{r}\left(N_{r}\right)\right)^{T}$ as the vector of $N_{r}$ redundant symbols. The time-domain signal in this first step is given by

$$
\mathbf{y}=\mathbf{F}_{N}^{-1} \mathbf{P}\left(\begin{array}{c}
\mathbf{x}_{d} \\
\mathbf{x}_{r}
\end{array}\right),
$$

where $\mathbf{F}_{N}$ is the $N \times N$ FFT matrix with $\left(\mathbf{F}_{N}\right)_{k, \ell}=$ $\frac{1}{\sqrt{N}} e^{-j 2 \pi \frac{k \ell}{N}}$ and $\mathbf{P}$ is the permutation matrix. This permutation matrix determines the positions of the data and redundant carriers. The $N \times N$ matrix $\mathbf{P}$ can be decomposed as $\mathbf{P}=\left[\begin{array}{ll}\mathbf{P}_{d} & \mathbf{P}_{r}\end{array}\right]$, where $\mathbf{P}_{d}$ is $N \times N_{d}$ and $\mathbf{P}_{r} N \times N_{r}$. The $N_{d}$ columns of $\mathbf{P}_{d}$ consist of the unit-weight vectors with the ' 1 ' at the positions $\tilde{n}_{\ell} \in I_{d}$, where $I_{d}$ is the set of $N_{d}$ data carrier positions. Similarly, the $N_{r}$ columns of $\mathbf{P}_{r}$ are the unit-weight vectors with the ' 1 ' at the positions $n_{\ell} \in I_{r}$, where $I_{r}$ is the set of $N_{r}$ redundant carrier positions.

We define the transform matrix $\mathbf{M}$ as $\mathbf{M}=\mathbf{F}_{N}^{-1} \mathbf{P}$. The transform matrix is decomposed as follows

$$
\mathbf{M}=\left(\begin{array}{ll}
\mathbf{M}_{11} & \mathbf{M}_{12} \\
\mathbf{M}_{21} & \mathbf{M}_{22}
\end{array}\right),
$$

where $\mathbf{M}_{11}$ is $\left(N-N_{u}\right) \times N_{d}, \mathbf{M}_{12}$ is $\left(N-N_{u}\right) \times N_{r}$, $\mathbf{M}_{21}$ is $N_{u} \times N_{d}$ and $\mathbf{M}_{22}$ is $N_{u} \times N_{r}$. The requirement that the last $N_{u}$ samples in the time domain must be zero, results in the set of linear equations $\mathbf{M}_{21} \mathbf{x}_{d}+\mathbf{M}_{22} \mathbf{x}_{r}=\mathbf{0}$. This implies the following relationship between the data symbols and redundant symbols ${ }^{2}$

$$
\mathbf{x}_{r}=-\mathbf{M}_{22}^{\dagger} \mathbf{M}_{21} \mathbf{x}_{d}=\mathbf{T} \mathbf{x}_{d}
$$

where $\mathbf{M}_{22}^{\dagger}=\mathbf{M}_{22}^{H}\left(\mathbf{M}_{22} \mathbf{M}_{22}^{H}\right)^{-1}$ is the Penrose-Moore pseudo-inverse. Hence, the time-domain signal $\mathbf{y}$ can be rewritten as

$$
\mathbf{y}=\mathbf{M}\left(\begin{array}{c}
\mathbf{I}_{N_{d}} \\
\mathbf{T}
\end{array}\right) \mathbf{x}_{d}=\mathbf{G} \mathbf{x}_{d}
$$

${ }^{2}$ When $N_{r}>N_{u}$, an infinite number of solutions exists for this underdetermined set of equations. However, it can easily be verified that the solution corresponding to the Penrose-Moore pseudo-inverse has the minimum average redundant symbol energy $E\left[\mathbf{x}_{r}^{H} \mathbf{x}_{r}\right]$. 

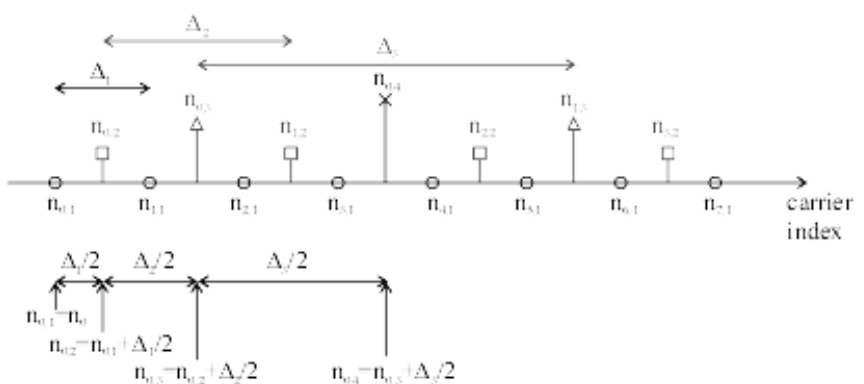

Fig. 2. Split redundant carrier distribution for $N_{r}=15: N_{r, 1}=8, N_{r, 2}=$ $4, N_{r, 3}=2, N_{r, 4}=1$. The spacings equal $\Delta_{1}=\frac{\Delta_{2}}{2}=\frac{\Delta_{3}}{4}$.

where $\mathbf{I}_{N_{d}}$ is the $N_{d} \times N_{d}$ identity matrix. In the matrix $\mathbf{G}$, the last $N_{u}$ rows are zero rows. by

The average energy of the time domain samples $\mathbf{y}$ is given

$$
P_{t}=E\left[\mathbf{y}^{H} \mathbf{y}\right]=P_{d}+P_{r}
$$

where $P_{d}=N_{d} E_{s}$ and $P_{r}=E_{s} \operatorname{trace}\left(\mathbf{T}^{H} \mathbf{T}\right)$ are the average energy of the data symbols and redundant symbols, respectively, and $E\left[x_{d}(i) x_{d}^{*}(j)\right]=E_{s} \delta_{i, j}$ is the energy of a data symbol. To obtain a high power efficiency, it is of importance that the average energy of the redundant symbols is as small as possible. Taking into account (3), the average energy of the redundant symbols can be rewritten as

$$
P_{r}=E_{s} \operatorname{trace}\left[\mathbf{M}_{21} \mathbf{M}_{21}^{H}\left(\mathbf{M}_{22} \mathbf{M}_{22}^{H}\right)^{-1}\right],
$$

where we have used the property $\operatorname{trace}(\mathbf{A B C})=\operatorname{trace}(\mathbf{C A B})$ [14]. Let us take a closer look at the matrices $\mathbf{M}_{21}$ and $\mathbf{M}_{22}$. The elements of these matrices are given by

$$
\begin{aligned}
& \left(\mathbf{M}_{21}\right)_{k, \ell}=\frac{1}{\sqrt{N}} e^{j 2 \pi \frac{k \tilde{n}_{\ell}}{N}} e^{-j 2 \pi \frac{N_{u} \tilde{n}_{\ell}}{N}} \quad \tilde{n}_{\ell} \in I_{d} \\
& \left(\mathbf{M}_{22}\right)_{k, \ell}=\frac{1}{\sqrt{N}} e^{j 2 \pi \frac{k n_{\ell}}{N}} e^{-j 2 \pi \frac{N_{u} n_{\ell}}{N}} \quad n_{\ell} \in I_{r}
\end{aligned}
$$

with $k=0, \ldots, N_{u}-1$. Hence, the matrices $\mathbf{M}_{21} \mathbf{M}_{21}^{H}$ and $\mathbf{M}_{22} \mathbf{M}_{22}^{H}$ from (6) are Hermitian Toeplitz matrices:

$$
\begin{aligned}
\left(\mathbf{M}_{21} \mathbf{M}_{21}^{H}\right)_{k, k^{\prime}} & =\frac{1}{N} \sum_{\ell=0}^{N_{d}-1} e^{j 2 \pi \frac{\left(k-k^{\prime}\right) \tilde{n}_{\ell}}{N}} \\
\left(\mathbf{M}_{22} \mathbf{M}_{22}^{H}\right)_{k, k^{\prime}} & =\frac{1}{N} \sum_{\ell=0}^{N_{r}-1} e^{j 2 \pi \frac{\left(k-k^{\prime}\right) n_{\ell}}{N}}
\end{aligned}
$$

where $k, k^{\prime}=0, \ldots, N_{u}-1$. Taking into account that $I_{r} \cup$ $I_{d}=\{0, \ldots, N-1\}$, it can easily be verified that $\mathbf{M}_{21} \mathbf{M}_{21}^{H}+$ $\mathbf{M}_{22} \mathbf{M}_{22}^{H}=\mathbf{I}_{N_{u}}$.

Let us first consider the special case where $N_{r}$ is a power of 2. In [7], the authors have shown through simulations that evenly spreading the redundant carriers over the bandwidth resulted in the minimum redundant energy. When $N_{r}$ is no longer a power of 2 , the situation becomes more complex. In [1], it is shown that the uniform distribution for the redundant carriers is no longer optimal, as in some cases, the matrix $\mathbf{M}_{22} \mathbf{M}_{22}^{H}$ becomes (close to) singular. In [1], a new distribution was introduced that resulted in essentially minimal average redundant energy. In this 'split' distribution, it is assumed that the number $N_{r}$ of redundant carriers is decomposed as a sum of powers of 2 :

$$
N_{r}=\sum_{\ell=1}^{L_{r}} N_{r, \ell},
$$

where $N_{r, \ell}=2^{x_{\ell}}, x_{\ell}$ is integer and $N_{r, 1}>N_{r, 2}>\ldots>$ $N_{r, L_{r}} \geq 1$. The set $I_{r}$ of $N_{r}$ redundant carriers is split in $L_{r}$ subsets $I_{r, \ell}$ with $N_{r, \ell}$ redundant carriers. Within each set $I_{r, \ell}$, the positions of the $N_{r, \ell}$ redundant carriers are uniformly distributed over the bandwidth with spacing $\Delta_{\ell}=\frac{N}{N_{r, \ell}}$, resulting in the carrier indices

$$
n_{m, \ell}=n_{0, \ell}+m \Delta_{\ell}, \quad m=0, \ldots, N_{r, \ell}-1 .
$$

The offsets $n_{0, \ell}$ of the carrier positions within the different sets $I_{r, \ell}$ equal

$$
\begin{aligned}
& n_{0,1}=n_{0} \\
& n_{0, \ell}=n_{0, \ell-1}+\frac{\Delta_{\ell-1}}{2}+m_{\ell} \Delta_{\ell-1}, \quad \ell>1,
\end{aligned}
$$

where $0 \leq n_{0}<\Delta_{1}$ and $m_{\ell}$ is integer ${ }^{3}$. In the special case when $m_{\ell}=\frac{N_{r, \ell-1}}{2 N_{r, \ell}}-1, \forall \ell>1$, the offsets $n_{0, \ell}$ reduce to $n_{0, \ell}=n_{0}+\frac{N}{2 N_{r, \ell}}-\frac{N}{2 N_{r, 1}}$. Figure 2 shows the split distribution for the case where $N_{r}^{r}=15$ with $m_{\ell}=0$. When $N_{r}$ is a power of 2 , it is clear that the proposed distribution reduces to a uniform distribution of the redundant carriers over the bandwidth.

Although we have assumed in this paper that all carriers are modulated, the proposed redundant carrier distribution can also be applied to systems containing a guard band with zero carriers. The only requirement is that the distribution must avoid these zero carriers. Typically, zero carriers are placed in the center of the frequency band and at the edges of the frequency band. As normally the number of zero carriers in the center of the frequency band is small, the main concern is therefore the zero carriers at the edges of the bandwidth. As long as the total number of zero carriers at the edges of the frequency band is smaller than the smallest spacing $\Delta_{1}=$ $\frac{N}{N_{r, 1}}$, it is possible to select an appropriate offset $n_{0}$ in order that none of the redundant carriers must be placed on a zero carrier. Fortunately, in most practical cases $N_{r}$ and thus $N_{r, 1}$ is much smaller than $N$, such that this spacing is sufficiently large to be able to avoid practical guard bands. In other words, given a number of zero carriers in the guard band, there will be a maximum on the number of redundant carriers that can be placed according to the proposed algorithm.

\section{Special CASE: $N_{u}=N_{r}$}

In this section, we consider the special case where the number of redundant carriers is the minimum possible, i.e. $N_{r}=N_{u}$. In this case, the matrices $\mathbf{M}_{11}$ and $\mathbf{M}_{22}$ in (2) are square matrices. With the split distribution, $\mathbf{M}_{11}$ and $\mathbf{M}_{22}$ are not singular, so they are invertible. It turns out that the redundant energy and total energy exhibit several symmetries as function of $N_{r}$.

\footnotetext{
${ }^{3}$ It can be verified that a shift of $n_{0, \ell}$ over a multiple of $\Delta_{\ell-1}$ does not influence the average redundant energy. This property might be interesting in a system with guard bands: in that way we might be able to avoid zero carriers.
} 
Proposition 1: The redundant energy is symmetrical in $N_{r}$ around $N / 2$, i.e.

$$
P_{r}\left(N_{r}\right)=P_{r}\left(N-N_{r}\right) .
$$

Proof: For the case of $N_{r}$ redundant carriers, we define the transform matrix $\mathbf{M}$, the set of redundant carriers $I_{r}$ and the set of data carriers $I_{d}$. For the case of $N-N_{r}$ redundant carriers, the transform matrix is $\tilde{M}$, and the sets of redundant carriers and data carriers are $\tilde{I}_{r}$ and $\tilde{I}_{d}$, respectively. Taking into account the distribution of the redundant carrier positions of the previous section, it can easily be verified that the positions of the data carriers also satisfy the same distribution but with $N_{d}=N-N_{r}$ positions instead of $N_{r}$ positions. Hence, the two cases with $N_{r}$ and $N-N_{r}$ redundant carriers can be interpreted as if the positions of the data carriers and redundant carriers are interchanged, i.e. $\tilde{I}_{d}=I_{r}$ and $\tilde{I}_{r}=I_{d}$. This implies the following relationship between the transform matrices $\mathbf{M}$ and $\tilde{\mathbf{M}}: \tilde{\mathbf{M}}_{11}=\mathbf{M}_{22} \operatorname{diag}\left\{e^{-j 2 \pi \frac{\left(N-N_{r}\right) n_{\ell}}{N}}\right\}, \tilde{\mathbf{M}}_{12}=$ $\mathbf{M}_{21} \operatorname{diag}\left\{e^{-j 2 \pi \frac{\left(N-N_{r}\right) \tilde{n}_{\ell}}{N}}\right\}, \tilde{\mathbf{M}}_{21}=\mathbf{M}_{12} \operatorname{diag}\left\{e^{j 2 \pi \frac{N_{r} n_{\ell}}{N}}\right\}$ and $\tilde{\mathbf{M}}_{22}=\mathbf{M}_{11} \operatorname{diag}\left\{e^{j 2 \pi \frac{N_{r} \tilde{n}_{\ell}}{N}}\right\}$, where $\operatorname{diag}\left\{y_{\ell}\right\}$ is a diagonal matrix with as diagonal elements $y_{\ell}$. Hence, the energy of the redundant carriers for the case with $N-N_{r}$ redundant carriers yields:

$$
\begin{aligned}
P_{r}\left(N-N_{r}\right) & =E_{s} \operatorname{trace}\left[\tilde{\mathbf{M}}_{21} \tilde{\mathbf{M}}_{21}^{H}\left(\tilde{\mathbf{M}}_{22} \tilde{\mathbf{M}}_{22}^{H}\right)^{-1}\right] \\
& =E_{s} \operatorname{trace}\left[\mathbf{M}_{12} \mathbf{M}_{12}^{H}\left(\mathbf{M}_{11} \mathbf{M}_{11}^{H}\right)^{-1}\right]
\end{aligned}
$$

Taking into account that $\mathbf{M}$ is a unitary matrix, i.e. $\mathbf{M M}^{H}=\mathbf{I}_{N}$, it follows that $\mathbf{M}_{12} \mathbf{M}_{22}^{H}+\mathbf{M}_{11} \mathbf{M}_{21}^{H}=\mathbf{0}$. As $\mathbf{M}_{11}$ and $\mathbf{M}_{22}$ are square invertible matrices, $\mathbf{M}_{12}=$ $-\mathbf{M}_{11} \mathbf{M}_{21}^{H}\left(\mathbf{M}_{22}^{H}\right)^{-1}$ such that (15) can be rewritten as

$$
\begin{aligned}
P_{r}\left(N-N_{r}\right) & =E_{s} \operatorname{trace}\left[\mathbf{M}_{11} \mathbf{M}_{21}^{H}\left(\mathbf{M}_{22} \mathbf{M}_{22}^{H}\right)^{-1}\right. \\
& \left.\mathbf{M}_{21} \mathbf{M}_{11}^{H}\left(\mathbf{M}_{11} \mathbf{M}_{11}^{H}\right)^{-1}\right] \\
& =E_{s} \operatorname{trace}\left[\mathbf{M}_{21} \mathbf{M}_{21}^{H}\left(\mathbf{M}_{22} \mathbf{M}_{22}^{H}\right)^{-1}\right] \\
& =P_{r}\left(N_{r}\right),
\end{aligned}
$$

where we have used trace $(\mathbf{A B C})=\operatorname{trace}(\mathbf{C A B})$ [14]. $\square$

Taking into account this symmetry and $P_{t}\left(N_{r}\right)=E_{s}(N-$ $\left.N_{r}\right)+P_{r}\left(N_{r}\right)$, it follows that

$$
P_{t}\left(N_{r}\right)=E_{s}\left(N-2 N_{r}\right)+P_{t}\left(N-N_{r}\right) .
$$

Proposition 2: The total energy is symmetrical in $N_{r}$ in the intervals $\left[N_{r, i}^{x}, N_{r, i}^{x}+\frac{N}{2^{i+1}}\right]$ around $N_{r, i}^{x}+\frac{N}{2^{i+2}}$, where $N_{r, i}^{x}=N \sum_{\ell=1}^{i} 2^{-\ell}=N-\frac{N}{2^{i}}$, for $i=0, \ldots, \log _{2} N-1$, i.e.

$$
P_{t}\left(N_{r, i}^{x}+x\right)=P_{t}\left(N_{r, i}^{x}+\frac{N}{2^{i+1}}-x\right), 0 \leq x \leq \frac{N}{2^{i+1}} .
$$

Proof: First, we show that

$$
P_{t}\left(\frac{N}{2^{i+1}}-x\right)=P_{t}(x)+2 E_{s}\left(2^{i}-1\right)\left(\frac{N}{2^{i+1}}-2 x\right)
$$

for $x \in\left[0, \frac{N}{2^{i+1}}\right], i=0, \ldots, \log _{2} N-1$.

When there are $x$ redundant carriers, there are $N_{d, 1}+N_{d, 2}$ data carriers, with $N_{d, 1}=N-\frac{N}{2^{i+1}}$ and $N_{d, 2}=\frac{N}{2^{i+1}}-x$. The transform matrix $\mathbf{M}$ in this case can be decomposed as

$$
\mathbf{M}=\left(\begin{array}{lll}
\mathbf{C}_{11} & \mathbf{C}_{12} & \mathbf{C}_{13} \\
\mathbf{C}_{21} & \mathbf{C}_{22} & \mathbf{C}_{23} \\
\mathbf{C}_{31} & \mathbf{C}_{32} & \mathbf{C}_{33}
\end{array}\right),
$$

where $\mathbf{C}_{j j^{\prime}}$ has dimension $d_{j} \times d_{j^{\prime}}$, where $\left\{d_{1}, d_{2}, d_{3}\right\}=$ $\left\{N_{d, 1}, N_{d, 2}, x\right\}$. Comparing (20) with (2), it follows that $\mathbf{M}_{21}=\left[\mathbf{C}_{31} \mathbf{C}_{32}\right]$ and $\mathbf{M}_{22}=\mathbf{C}_{33}$.

When there are $\frac{N}{2^{2+1}}-x$ redundant carriers, the transform matrix can be written as

$$
\tilde{\mathbf{M}}=\left(\begin{array}{lll}
\tilde{\mathbf{C}}_{11} & \tilde{\mathbf{C}}_{12} & \tilde{\mathbf{C}}_{13} \\
\tilde{\mathbf{C}}_{21} & \tilde{\mathbf{C}}_{22} & \tilde{\mathbf{C}}_{23} \\
\tilde{\mathbf{C}}_{31} & \tilde{\mathbf{C}}_{32} & \tilde{\mathbf{C}}_{33}
\end{array}\right),
$$

where $\tilde{\tilde{C}}_{j j^{\prime}}$ has dimension $\tilde{d}_{j} \quad \underset{\tilde{d}_{1}}{\tilde{d}_{j^{\prime}}}, \quad$ where $\left\{\tilde{d}_{1}, \tilde{d}_{2}, \tilde{d}_{3}\right\}=\left\{N_{d, 1}, x, N_{d, 2}\right\}$. Similarly, $\tilde{\mathbf{M}}_{21}=\left[\tilde{\mathbf{C}}_{31} \tilde{\mathbf{C}}_{32}\right]$ and $\tilde{\mathbf{M}}_{22}=\tilde{\mathbf{C}}_{33}$. The relationship between the submatrices of $\mathbf{M}$ and $\tilde{\mathbf{M}}$ is given by $\tilde{\mathbf{C}}_{\ell, m}=\mathbf{C}_{z(\ell), z(m)} \mathbf{D}_{z(\ell), z(m)}$, where $\{z(1), z(2), z(3)\}=\{1,3,2\}, \mathbf{D}_{\ell, m}=\operatorname{diag}\left\{e^{j 2 \pi \frac{x_{\ell} y_{m}}{N}}\right\}$, $\left\{x_{1}, x_{2}, x_{3}\right\}=\left\{0,-d_{2}, d_{3}\right\}$ and $\left\{y_{1}, y_{2}, y_{3}\right\}=$ $\left\{\tilde{n}_{\ell, 1}, n_{\ell}, \tilde{n}_{\ell, 2}\right\}$. The carrier indices $\tilde{n}_{\ell, 1}$ and $\tilde{n}_{\ell, 2}$ correspond to the $N_{d, 1}$ and $N_{d, 2}$ data carriers, and $n_{\ell}$ to the $x$ redundant carriers from case 1 , respectively.

It can easily be verified that $\mathbf{C}_{21} \mathbf{C}_{21}^{H}=\frac{N_{d, 1}}{N} \mathbf{I}_{\tilde{x}}, \mathbf{C}_{31} \mathbf{C}_{31}^{H}=$ $\frac{N_{d, 1}}{N} \mathbf{I}_{x}$ and $\mathbf{C}_{21} \mathbf{C}_{31}^{H}=\mathbf{0}$ where $\tilde{x}=\frac{N}{2^{i+1}}-x=N_{d, 2}$. Hence, the total energy in the two cases can be written as

$$
\begin{aligned}
P_{t}(x) & =E_{s}(N-x) \\
& +E_{s} \operatorname{trace}\left[\left(\frac{N_{d, 1}}{N} \mathbf{I}_{x}+\mathbf{C}_{32} \mathbf{C}_{32}^{H}\right)\left(\mathbf{C}_{33} \mathbf{C}_{33}^{H}\right)^{-1}\right] \\
& =E_{s}(N-x) \\
& +E_{s} \alpha \operatorname{trace}\left(\mathbf{C}_{33} \mathbf{C}_{33}^{H}\right)^{-1} \\
& +E_{s} \operatorname{trace}\left[\mathbf{C}_{32} \mathbf{C}_{32}^{H}\left(\mathbf{C}_{33} \mathbf{C}_{33}^{H}\right)^{-1}\right] \\
P_{t}(\tilde{x}) & =E_{s}(N-\tilde{x}) \\
& +E_{s} \operatorname{trace}\left[\left(\frac{N_{d, 1}}{N} \mathbf{I}_{\tilde{x}}+\mathbf{C}_{23} \mathbf{C}_{23}^{H}\right)\left(\mathbf{C}_{22} \mathbf{C}_{22}^{H}\right)^{-1}\right] \\
& =E_{s}(N-\tilde{x}) \\
& +E_{s} \alpha \operatorname{trace}\left(\mathbf{C}_{22} \mathbf{C}_{22}^{H}\right)^{-1} \\
& +E_{s} \operatorname{trace}\left[\mathbf{C}_{23} \mathbf{C}_{23}^{H}\left(\mathbf{C}_{22} \mathbf{C}_{22}^{H}\right)^{-1}\right]
\end{aligned}
$$

where $\alpha=1-2^{-(i+1)}$. Because of the unitary character of the matrix $\mathbf{M}$, i.e. $\mathbf{M} \mathbf{M}^{H}=\mathbf{I}_{N}$, it follows that $\sum_{i=1}^{3} \mathbf{C}_{i j} \mathbf{C}_{i j}^{H}=$ $\mathbf{I}_{\tilde{d}_{j}}, j=1,2,3$ and $\sum_{i=1}^{3} \mathbf{C}_{j i} \mathbf{C}_{k i}^{H}=\mathbf{0}$, with $j, k=1,2,3$ and $j \neq k$. Using $\mathbf{C}_{21} \mathbf{C}_{21}^{H}=\frac{N_{d, 1}}{N} \mathbf{I}_{\tilde{x}}, \mathbf{C}_{31} \mathbf{C}_{31}^{H}=\frac{N_{d, 1}}{N} \mathbf{I}_{x}$ and $\mathbf{C}_{21} \mathbf{C}_{31}^{H}=\mathbf{0}$, this implies $\mathbf{C}_{22} \mathbf{C}_{22}^{H}+\mathbf{C}_{23} \mathbf{C}_{23}^{H}=\frac{1}{2^{i+1}} \mathbf{I}_{\tilde{x}}$, $\mathbf{C}_{32} \mathbf{C}_{32}^{H}+\mathbf{C}_{33} \mathbf{C}_{33}^{H}=\frac{1}{2^{i+1}} \mathbf{I}_{x}$ and $\mathbf{C}_{22} \mathbf{C}_{32}^{H}+\mathbf{C}_{23} \mathbf{C}_{33}^{H^{2}}=\mathbf{0}$. Rewriting these relations yield

$$
\begin{aligned}
\frac{1}{2^{i+1}}\left(\mathbf{C}_{22} \mathbf{C}_{22}^{H}\right)^{-1} & =\mathbf{I}_{\tilde{x}}+\mathbf{C}_{23} \mathbf{C}_{23}^{H}\left(\mathbf{C}_{22} \mathbf{C}_{22}^{H}\right)^{-1} \\
\frac{1}{2^{i+1}}\left(\mathbf{C}_{33} \mathbf{C}_{33}^{H}\right)^{-1} & =\mathbf{I}_{x}+\mathbf{C}_{32} \mathbf{C}_{32}^{H}\left(\mathbf{C}_{33} \mathbf{C}_{33}^{H}\right)^{-1} \\
\mathbf{C}_{23} & =-\mathbf{C}_{22} \mathbf{C}_{32}^{H}\left(\mathbf{C}_{33}^{H}\right)^{-1} .
\end{aligned}
$$

Substitution in the total energy from (22) and (23) yields

$$
\begin{aligned}
P_{t}(x) & =E_{s}\left(N+2\left(2^{i}-1\right) x\right) \\
& +E_{s} 2^{i+1} \operatorname{trace}\left[\mathbf{C}_{32} \mathbf{C}_{32}^{H}\left(\mathbf{C}_{33} \mathbf{C}_{33}^{H}\right)^{-1}\right] \\
P_{t}(\tilde{x}) & =E_{s}\left(N+2\left(2^{i}-1\right) \tilde{x}\right) \\
& +E_{s} 2^{i+1} \operatorname{trace}\left[\mathbf{C}_{23} \mathbf{C}_{23}^{H}\left(\mathbf{C}_{22} \mathbf{C}_{22}^{H}\right)^{-1}\right]
\end{aligned}
$$




$$
\begin{aligned}
& =E_{s}\left(N+2\left(2^{i}-1\right) \tilde{x}\right) \\
& +\quad E_{s} 2^{i+1} \text { trace }\left[\mathbf{C}_{32} \mathbf{C}_{32}^{H}\left(\mathbf{C}_{33} \mathbf{C}_{33}^{H}\right)^{-1}\right] .
\end{aligned}
$$

Comparing these two expressions, and taking into account that $\tilde{x}=\frac{N}{2^{i+1}}-x$, it follows that

$$
P_{t}\left(\frac{N}{2^{i+1}}-x\right)=P_{t}(x)+2 E_{s}\left(2^{i}-1\right)\left(\frac{N}{2^{i+1}}-2 x\right) .
$$

In the second step, we show that $P_{t}\left(N_{r, i}^{x}+x\right)=P_{t}\left(N_{r, i}^{x}+\right.$ $\tilde{x})$, i.e. that the total energy is symmetric as function of $N_{r}$ in the interval $\left[N_{r, i}^{x}, N_{r, i}^{x}+\frac{N}{2^{i+1}}\right]$, where $N_{r, i}^{x}=N-\frac{N}{2^{i}}, \tilde{x}=$ $\frac{N}{2^{i+1}}-x$ and $x \in\left[0, \frac{N}{2^{i+1}}\right]$.

The total energy $P_{t}\left(N_{r, i}^{x}+x\right)$ can be rewritten as

$$
\begin{aligned}
P_{t}\left(N_{r, i}^{x}+x\right) & =P_{t}\left(m_{x, i}\right)-E_{s}\left(N-2 m_{x, i}\right) \\
& =P_{t}(x)-2 x\left(2^{i}-1\right) E_{s},
\end{aligned}
$$

where $m_{x, i}=\frac{N}{2^{i}}-x$. We have used (17) and (27) to obtain the first and second line in (28), respectively. The total energy $P_{t}\left(N_{r, i}^{x}+\tilde{x}\right)$ equals

$$
\begin{aligned}
P_{t}\left(N_{r, i}^{x}+\tilde{x}\right) & =P_{t}(\tilde{x})-2 \tilde{x}\left(2^{i}-1\right) E_{s} \\
& =P_{t}(x)-2 x\left(2^{i}-1\right) E_{s} \\
& =P_{t}\left(N_{r, i}^{x}+x\right) .
\end{aligned}
$$

where the first line follows from (28) and the second line from (27). $\square$

It can easily be verified from (5) that $P_{t}(0)=N E_{s}$ and $P_{t}(1)=2(N-1) E_{s}$. Based on these initial values for the total energy and the symmetries in the total and redundant energies, a recursive relation for the total energy for all other values of $N_{r}$ can be derived (see the appendix):

$$
P_{t}\left(2^{y}+Y\right)=P_{t}(Y)+E_{s}\left(N-2^{y+1}-\frac{N}{2^{y}} Y\right)
$$

where $0 \leq y \leq \log _{2} N-1$ and $Y<2^{y}$. Taking into account that $N_{r}=\sum_{i=1}^{L} N_{r, i}=\sum_{i=1}^{L} 2^{x_{i}}$, this recursive relation can be written after some straightforward manipulations as a closed expression for the total energy:

$$
\begin{aligned}
P_{t}\left(N_{r}\right) & =E_{s}\left(N-2 N_{r}\right) \\
& +E_{s} \sum_{i=1}^{L_{r}}\left(N-\frac{N}{N_{r, i}} \sum_{\ell=i+1}^{L_{r}} N_{r, \ell}\right) .
\end{aligned}
$$

\section{General CASE: $N_{u} \leq N_{r}$}

When $N_{r}>N_{u}$, the matrix $\mathbf{M}_{22}$ is no longer a square matrix. In this case, a profound analysis of the average redundant energy can be achieved by evaluating the eigenvalues of the matrices $\mathbf{M}_{21} \mathbf{M}_{21}^{H}$ and $\mathbf{M}_{22} \mathbf{M}_{22}^{H}$. Assuming the eigenvalues and eigenvectors of the matrix $\mathbf{M}_{22} \mathbf{M}_{22}^{H}$ are given by $\lambda_{m}$ and $\mathbf{v}_{m}, m=1, \ldots, N_{u}$, respectively, it follows from $\mathbf{M}_{21} \mathbf{M}_{21}^{H}+\mathbf{M}_{22} \mathbf{M}_{22}^{H}=\mathbf{I}_{N_{u}}$ that the eigenvalues and eigenvectors of $\mathbf{M}_{21} \mathbf{M}_{21}^{H}$ are $1-\lambda_{m}$ and $\mathbf{v}_{m}, m=1, \ldots, N_{u}$, respectively. Taking this into account, the average redundant energy (6) can be rewritten as

$$
P_{r}=E_{s} \sum_{m=1}^{N_{u}} \frac{1-\lambda_{m}}{\lambda_{m}}
$$

As $\mathbf{M}_{22} \mathbf{M}_{22}^{H}$ is a Hermitian matrix, its eigenvalues are real valued. Further, the matrix $\mathbf{M}_{22} \mathbf{M}_{22}^{H}$ is positive semi-definite:

$$
\mathbf{x}^{H} \mathbf{M}_{22} \mathbf{M}_{22}^{H} \mathbf{x}=\sum_{\ell=0}^{N_{r}-1}\left|\frac{1}{\sqrt{N}} \sum_{k=0}^{N_{u}-1} x_{k} e^{-j 2 \pi \frac{k n_{\ell}}{N}}\right| \geq 0,
$$

such that the eigenvalues are positive: $\lambda_{m} \geq 0$. Similarly, $\mathbf{M}_{21} \mathbf{M}_{21}^{H}$ is positive semi-definite, such that $1-\lambda_{m} \geq 0$. Hence, the eigenvalues $\lambda_{m}$ are restricted to the interval $0 \leq$ $\lambda_{m} \leq 1$.

As trace $\left(\mathbf{M}_{22} \mathbf{M}_{22}^{H}\right)=\frac{N_{r} N_{u}}{N}$, the sum of the eigenvalues equals $\sum_{\ell=1}^{N_{u}} \lambda_{m}=\frac{N_{r} N_{u}}{N}$. Taking into account (32), it can easily be verified that the average redundant energy is minimized when all eigenvalues are equal and given by $\lambda_{m}=\frac{N_{r}}{N}$. It can easily be shown that this implies that the matrix $\mathbf{M}_{22} \mathbf{M}_{22}^{H}$ should be a diagonal matrix: $\mathbf{M}_{22} \mathbf{M}_{22}^{H}=\frac{N_{r}}{N} \mathbf{I}_{N_{u}}$. In that case, the redundant energy equals $P_{r, \min }=E_{s} \frac{N_{u} N_{d}}{N_{r}}$. In the special case where $N_{r}$ is a power of 2 and the redundant carriers are uniformly distributed over the bandwidth ${ }^{4}$, the matrix $\mathbf{M}_{22} \mathbf{M}_{22}^{H}$ reduces to $\mathbf{M}_{22} \mathbf{M}_{22}^{H}=\frac{N_{r}}{N} \mathbf{I}_{N_{u}}$ : the average redundant energy equals $P_{r}=P_{r, \min }$. Hence, this confirms the results from [7] where the authors have shown through simulations that a (close to) uniform distribution was optimal for $N_{r}$ equal to a power of 2 . This implies that the total energy to construct the data part of the UW-OFDM block equals $P_{t}=N_{d} E_{s}\left(1+\frac{N_{u}}{N_{r}}\right)$. In case $N_{u}=N_{r}$, the extra energy needed for UW-OFDM to create a $N_{u}$ length zero interval in the time domain equals the energy needed for the data symbols: UW-OFDM doubles the energy needed to transmit $N_{d}$ data symbols. This does not yet take into account the energy needed for the unique word! So at a first sight, UWOFDM is very energy inefficient. However, this extra energy is used to create redundancy in the signal, as in an error correcting code. At the receiver, using a data detector that fully exploits all redundancy available in the signal will transform this extra energy into a sort of 'coding gain', such that the performance of UW-OFDM is comparable to standard OFDM techniques.

Note that when one or more of the eigenvalues $\lambda_{m}$ is small as compared to the other eigenvalues, the redundant energy (32) can strongly increase. In the following, we evaluate the eigenvalues $\lambda_{m}$ from $\mathbf{M}_{22} \mathbf{M}_{22}^{H}$. We rewrite the matrix $\mathbf{M}_{22} \mathbf{M}_{22}^{H}$ as

$$
\mathbf{M}_{22} \mathbf{M}_{22}^{H}=\frac{1}{N} \boldsymbol{\Theta}^{H} \mathbf{A} \boldsymbol{\Theta}
$$

where $\boldsymbol{\Theta}=\operatorname{diag}\left\{e^{j 2 \pi \frac{k n_{0}}{N}}\right\}, k=0, \ldots, N_{u}-1$ and $\mathbf{A}$ is a real-valued symmetric Toeplitz matrix with as first row the vector $\left[a(0) \ldots a\left(N_{u}-1\right)\right]$ with

$$
a(n)=\sum_{\ell=1}^{L_{r}} N_{r, \ell} \sum_{m=0}^{M_{r, \ell}}(-1)^{m} \delta\left(n-m N_{r, \ell}\right),
$$

where $N_{u}=M_{r, \ell} N_{r, \ell}+K_{r, \ell}$, and $0 \leq K_{r, \ell}<N_{r, \ell}$. Taking into account that the matrix $\Theta$ is a unitary matrix, the evaluation of the eigenvalues of $\mathbf{M}_{22} \mathbf{M}_{22}^{H}$ reduces to the

\footnotetext{
${ }^{4}$ Note that in this paper we have restricted our attention to $N$ equal to a power of 2. In the case where $N$ is not a power of 2, every $N_{r}$ that is a factor of $N$ combined with a uniform distribution of the redundant carriers yields $\mathbf{M}_{22} \mathbf{M}_{22}^{H}=\frac{N_{r}}{N} \mathbf{I}_{N_{u}}$ and hence minimum average redundant energy.
} 
search for the eigenvalues of $\mathbf{A}$. Note that as $\mathbf{A}$ is independent of $N$, also its eigenvalues will be independent of $N$.

Let us define $[x]_{\leq}=\arg \min _{m}\left\{x \geq N_{r, m} \mid N_{r, m} \in J_{r}\right\}$, with $J_{r}=\left\{N_{r, 1}, \ldots, N_{r, L_{r}}\right\}$, i.e. $[x]_{\leq}$is the index of the largest value in $J_{r}$ that is equal to or smaller than $x$. Further, the $m \times m$ Hadamard matrix $\mathbf{H}_{m}$ can be constructed in the following recursive way:

$$
\mathbf{H}_{2 m}=\frac{1}{\sqrt{2}}\left(\begin{array}{cc}
\mathbf{H}_{m} & \mathbf{H}_{m} \\
\mathbf{H}_{m} & -\mathbf{H}_{m}
\end{array}\right)
$$

with $\mathbf{H}_{1}=1$. The Hadamard matrix $\mathbf{H}_{m}$ is a symmetric unitary matrix.

Let us first consider the special case where $N_{u}$ is a power of 2. In that case, it can be verified that the Hadamard matrix $\mathbf{H}_{N_{u}}$ diagonalizes the matrix $\mathbf{A}: \mathbf{H}_{N_{u}} \mathbf{A} \mathbf{H}_{N_{u}}=\operatorname{diag}\left\{\lambda_{k}\right\}$, $k=1, \ldots, N_{u}$. The eigenvalues of $\mathbf{A}$ in that case are $\lambda=$ $\sum_{\ell=1}^{\left[N_{u}\right]} \leq N_{r, \ell}$ with multiplicity $\mu$ and $\lambda=\sum_{\ell=1}^{\left[N_{u}\right]} \leq N_{r, \ell}+N_{u}$ with multiplicity $N_{u}-\mu$, where $\mu$ is given by

$$
\mu= \begin{cases}N_{u}-\sum_{\ell=\left[N_{u} / 2\right]_{\leq}}^{L_{r}} N_{r, \ell} & N_{u}>N_{r,\left[N_{u}\right]_{\leq}} \\ \sum_{\ell=\left[N_{u} / 2\right]_{\leq}}^{L_{r}} N_{r, \ell} & N_{u} \leq N_{r,\left[N_{u}\right]_{\leq}}\end{cases}
$$

When $N_{u}$ is not a power of 2, the following strategy can be used to determine the eigenvalues of $\mathbf{A}$. First, we decompose $N_{u}$ into a sum of powers of 2 :

$$
N_{u}=\sum_{\ell=1}^{L_{u}} N_{u, \ell}
$$

with $N_{u, \ell}>N_{u, \ell+1}, N_{u, \ell}=2^{x_{\ell}}$ and $x_{\ell}$ integer. Further, we decompose the matrix $\mathbf{A}$ as

$$
\mathbf{A}=\left(\begin{array}{cc}
\boldsymbol{\alpha}_{1} & \boldsymbol{\beta}_{1} \\
\boldsymbol{\beta}_{1}^{T} & \gamma_{1}
\end{array}\right)
$$

with $\boldsymbol{\alpha}_{1}$ is $N_{u, 1} \times N_{u, 1}, \boldsymbol{\beta}_{1}$ is $N_{u, 1} \times\left(N_{u}-N_{u, 1}\right)$ and $\boldsymbol{\gamma}_{1}$ is $\left(N_{u}-N_{u, 1}\right) \times\left(N_{u}-N_{u, 1}\right)$. The characteristic equation of $\mathbf{A}$, determining the eigenvalues, is given by $\operatorname{det}\left(\mathbf{A}-\lambda \mathbf{I}_{\mathbf{N}_{\mathbf{u}}}\right)=0$. This equation can be rewritten as [14]

$$
\begin{aligned}
& \operatorname{det}\left(\mathbf{A}-\lambda \mathbf{I}_{N_{u}}\right)=\operatorname{det}\left(\boldsymbol{\alpha}_{1}-\lambda \mathbf{I}_{N_{u, 1}}\right) \\
& \quad \cdot \operatorname{det}\left(\boldsymbol{\gamma}_{1}-\lambda \mathbf{I}_{N_{u, 1}}-\boldsymbol{\beta}_{1}^{T}\left(\boldsymbol{\alpha}_{1}-\lambda \mathbf{I}_{N_{u, 1}}\right)^{-1} \boldsymbol{\beta}_{1}\right) .
\end{aligned}
$$

The matrix $\boldsymbol{\alpha}_{1}$ can be diagonalized by multiplying it with the unitary Hadamard matrix $\mathbf{H}_{N_{u, 1}}$, i.e. $\boldsymbol{\Lambda}_{1}=\mathbf{H}_{N_{u, 1}} \boldsymbol{\alpha}_{1} \mathbf{H}_{N_{u, 1}}$. The eigenvalues of $\boldsymbol{\alpha}_{1}$, i.e. the diagonal elements of $\boldsymbol{\Lambda}_{1}$ are $\rho_{1}=\sum_{\ell=1}^{\left[N_{u, 1}\right]} \leq N_{r, \ell}$ with multiplicity $\nu_{1}$ and $\rho_{1}+N_{u, 1}$ with multiplicity $N_{u, 1}-\nu_{1}$, where

$$
\nu_{1}= \begin{cases}N_{u, 1}-\sum_{\ell=\left[N_{u, 1} / 2\right]_{\leq}}^{L_{r}} N_{r, \ell} & N_{u}>N_{r,\left[N_{u}\right]_{\leq}} . \\ \sum_{\ell=\left[N_{u, 1} / 2\right]_{\leq}}^{L_{r}} N_{r, \ell} & N_{u} \leq N_{r,\left[N_{u}\right]_{\leq}}\end{cases}
$$

Further, the product $\boldsymbol{\beta}_{1}^{T}\left(\boldsymbol{\alpha}_{1}-\lambda \mathbf{I}_{N_{u, 1}}\right)^{-1} \boldsymbol{\beta}_{1}$ can be rewritten as

$$
\begin{aligned}
\boldsymbol{\beta}_{1}^{T}\left(\boldsymbol{\alpha}_{1}-\lambda \mathbf{I}_{N_{u, 1}}\right)^{-1} \boldsymbol{\beta}_{1} & =\boldsymbol{\beta}_{1}^{T} \mathbf{H}_{N_{u}, 1}\left(\boldsymbol{\Lambda}_{1}-\lambda \mathbf{I}_{N_{u, 1}}\right)^{-1} \mathbf{H}_{N_{u}, 1} \boldsymbol{\beta}_{1} \\
& =\frac{1}{\epsilon_{1}-\lambda} \boldsymbol{\beta}_{1}^{T} \boldsymbol{\beta}_{1}
\end{aligned}
$$

where

$$
\epsilon_{1}= \begin{cases}\rho_{1}+N_{u, 1} & N_{u}>N_{r,\left[N_{u}\right]_{\leq}} \\ \rho_{1} & N_{u} \leq N_{r,\left[N_{u}\right]_{\leq}}\end{cases}
$$

The second line in (42) follows from the fact that $\mathbf{H}_{N_{u, 1}} \boldsymbol{\beta}_{1}$ has zero rows where $\boldsymbol{\Lambda}_{1}$ has diagonal element different from $\epsilon_{1}$, and non-zero rows where the diagonal element of $\boldsymbol{\Lambda}_{1}$ equals $\epsilon_{1}$. Hence, the matrix $\left(\boldsymbol{\Lambda}_{1}-\lambda \mathbf{I}_{N_{u, 1}}\right)^{-1}$ can be replaced by the matrix $\frac{1}{\epsilon_{1}-\lambda} \mathbf{I}_{N_{u, 1}}$. Further, it can easily be verified that the product $\boldsymbol{\beta}_{1}^{T} \boldsymbol{\beta}_{1}$ equals

$$
\boldsymbol{\beta}_{1}^{T} \boldsymbol{\beta}_{1}= \begin{cases}N_{u, 1}\left(\gamma_{1}-\rho_{1} \mathbf{I}_{\bar{N}_{u, 1}}\right) & N_{u}>N_{r,\left[N_{u}\right]_{\leq}} \\ N_{u, 1}\left(\left(\rho_{1}+N_{u, 1}\right) \mathbf{I}_{\bar{N}_{u, 1}}-\gamma_{1}\right) & N_{u} \leq N_{r,\left[N_{u}\right]_{\leq}}\end{cases}
$$

where $\bar{N}_{u, i}=N_{u}-\sum_{\ell=1}^{i} N_{u, \ell}$. Hence, the second factor in (40) can be rewritten as

$$
\begin{aligned}
& \operatorname{det}\left(\boldsymbol{\gamma}_{1}-\lambda \mathbf{I}_{N_{u, 1}}-\boldsymbol{\beta}_{1}^{T}\left(\boldsymbol{\alpha}_{1}-\lambda \mathbf{I}_{N_{u, 1}}\right)^{-1} \boldsymbol{\beta}_{1}\right)= \\
& \left\{\begin{array}{r}
\operatorname{det}\left[\frac{\rho_{1}-\lambda}{\rho_{1}+N_{u, 1}-\lambda}\left(\gamma_{1}-\left(\lambda-N_{u, 1}\right) \mathbf{I}_{\bar{N}_{u, 1}}\right)\right], \\
N_{u}>N_{r,\left[N_{u}\right]_{\leq}} \\
\operatorname{det}\left[\frac{\rho_{1}+N_{u, 1}-\lambda}{\rho_{1}-\lambda}\left(\gamma_{1}-\left(\lambda+N_{u, 1}\right) \mathbf{I}_{\bar{N}_{u, 1}}\right)\right], \\
N_{u} \leq N_{r,\left[N_{u}\right]_{\leq}}
\end{array}\right.
\end{aligned}
$$

As the structure of $\gamma_{1}$ is the same as the structure of $\mathbf{A}$, this suggests that the eigenvalues of $\mathbf{A}$ can be calculated in a recursive way.

Let us define the block matrix

$$
\gamma_{i-1}=\left(\begin{array}{cc}
\boldsymbol{\alpha}_{i} & \boldsymbol{\beta}_{i} \\
\boldsymbol{\beta}_{i}^{T} & \gamma_{i}
\end{array}\right)
$$

where $\gamma_{0}=\mathbf{A}, \boldsymbol{\alpha}_{i}$ is $N_{u, i} \times N_{u, i}, \boldsymbol{\beta}_{i}$ is $N_{u, i} \times \bar{N}_{u, i}$ and $\gamma_{i}$ is $\bar{N}_{u, i} \times \bar{N}_{u, i}$. The matrix $\boldsymbol{\alpha}_{i}$ can be diagonalized with the Hadamard matrix $\mathbf{H}_{N_{u, i}}$, yielding the eigenvalues of $\boldsymbol{\alpha}_{i} \lambda=\rho_{i}$ with multiplicity $\nu_{i}$ and $\lambda=\rho_{i}+N_{u, i}$ with multiplicity $N_{u, i}-\nu_{i}$, where $\rho_{i}=\sum_{\ell=1}^{\left[N_{u, i}\right] \leq} N_{r, \ell}$ and $\nu_{i}=N_{u, i}-\sum_{\ell=\left[N_{u, i} / 2\right]_{\leq}}^{L_{u}} N_{u, \ell}$. When $N_{u}>N_{r,\left[N_{u}\right]_{\leq}}$, the recursive equation yields

$$
\begin{aligned}
& \operatorname{det}\left(\boldsymbol{\gamma}_{i-1}-\lambda_{i} \mathbf{I}_{\bar{N}_{u, i}}\right)= \\
& \quad\left(\rho_{i}-\lambda_{i}\right)^{\nu_{i}+\bar{N}_{u, i}}\left(\rho_{i}+N_{u, i}-\lambda_{i}\right)^{N_{u, i}-\bar{N}_{u, i}-\nu_{i}} \\
& \cdot \operatorname{det}\left(\gamma_{i}-\lambda_{i+1} \mathbf{I}_{\bar{N}_{u, i+1}}\right)
\end{aligned}
$$

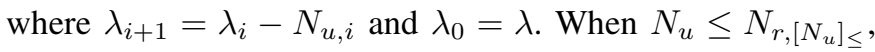
the recursive equation yields

$$
\begin{aligned}
& \operatorname{det}\left(\gamma_{i-1}-\lambda_{i} \mathbf{I}_{\bar{N}_{u, i}}\right)= \\
& \left(\rho_{i}-\lambda_{i}\right)^{\nu_{i}-\bar{N}_{u, i}}\left(\rho_{i}+N_{u, i}-\lambda_{i}\right)^{N_{u, i}+\bar{N}_{u, i}-\nu_{i}} \\
& \cdot \operatorname{det}\left(\gamma_{i}-\lambda_{i+1} \mathbf{I}_{\bar{N}_{u, i+1}}\right)
\end{aligned}
$$

where $\lambda_{i+1}=\lambda_{i}+N_{u, i}$. Note that in the first case, the eigenvalues of $\mathbf{A}$ are $\rho_{i}+\sum_{\ell=1}^{i-1} N_{u, \ell}$ and $\rho_{i}+\sum_{\ell=1}^{i} N_{u, \ell}$ with multiplicities $\nu_{i}+\bar{N}_{u, i}$ and $N_{u, i}-\bar{N}_{u, i}-\nu_{i}$, respectively, and in the second case $\rho_{i}-\sum_{\ell=1}^{i-1} N_{u, \ell}$ and $\rho_{i}+N_{u, i}-\sum_{\ell=1}^{i-1} N_{u, \ell}$ with multiplicities $\nu_{i}-\bar{N}_{u, i}$ and $N_{u, i}+\bar{N}_{u, i}-\nu_{i}$, respectively. In both cases, it turns out that the eigenvalues of $\mathbf{A}$ are integer values.

Let us consider the special case $N_{u}=N_{r}$. In this case, $N_{u, \ell}=N_{r, \ell}$, such that $\rho_{i}=\sum_{\ell=1}^{i} N_{u, \ell}$ and $\nu_{i}=N_{u, i}-$ $\sum_{\ell=i+1}^{L_{u}} N_{u, \ell}$. Hence, the eigenvalues of $\mathbf{A}$ are given by $\lambda=N_{u, i}$ with multiplicity $\nu_{i}-\bar{N}_{u, i}=N_{u, i}-2 \sum_{\ell=i+1}^{L_{u}} N_{u, \ell}$ and $\lambda=2 N_{u, i}$ with multiplicity $2 \sum_{\ell=i+1}^{L_{u}} N_{u, \ell}$, for $i=$ 
$1, \ldots, L_{u}$. The energy of the redundant carriers is then given by

$$
P_{r}=E_{s} \sum_{i=1}^{L_{u}}\left(N-N_{u, i}-\frac{N}{N_{u, i}} \sum_{\ell=i+1}^{L_{u}} N_{u, \ell}\right)
$$

Taking into account hat $P_{d}=\left(N-N_{r}\right) E_{s}$, this expression leads to the expression (31) for the total energy, obtained in the previous section.

When $N_{r}>N_{u}$, in general no closed expression for the redundant energy can be derived. Hence, the eigenvalues should be calculated for this case, and based on these eigenvalues, the redundant energy can be computed. However, for some special cases, a closed form expression for the redundant energy can be found. When $N_{r}=2^{m}-1$, the eigenvalues of $\mathbf{A}$ are $\lambda=2^{m}-N_{u}$ with multiplicity $\nu=1$ and $\lambda=2^{m}$ with multiplicity $\nu=N_{u}-1$, resulting in the redundant energy

$$
P_{r}=E_{s}\left(\frac{N-2^{m}+N_{u}}{2^{m}-N_{u}}+\left(N_{u}-1\right) \frac{N-2^{m}}{2^{m}}\right) .
$$

When $N_{r}=2^{m}+1$ and $N_{u} \leq 2^{m}$, the eigenvalues are $\lambda=$ $2^{m}+N_{u}$ with multiplicity $\nu=1$ and $\lambda=2^{m}$ with multiplicity $\nu=N_{u}-1$, resulting in the redundant energy

$$
P_{r}=E_{s}\left(\frac{N-2^{m}-N_{u}}{2^{m}+N_{u}}+\left(N_{u}-1\right) \frac{N-2^{m}}{2^{m}}\right) \text {. }
$$

Although in the previous paragraphs, we have derived a method to effectively calculate the eigenvalues of the matrix A, it still requires computation time. It might be of interest to obtain bounds for the minimum and maximum eigenvalues of A that can be computed at no cost. In the following, we give some bounds for the minimum and maximum eigenvalues that can directly be calculated from $N_{u}$ and $N_{r}$. This might be a valuable tool in the design of an UW-OFDM system.

Proposition 3: The eigenvalues of $\mathbf{A}$ are within the interval $\left[\lambda_{\min }, \lambda_{\max }\right]$, where $\lambda_{\min }=\sum_{\ell=1}^{\left[2 N_{u, 1}\right]_{\leq}} N_{r, \ell}$ and $\lambda_{\max }=$ $\lambda_{\min }+2 N_{u, 1}$. Further, the minimum eigenvalue of $\mathbf{A}$ is upper limited by $\tilde{\lambda}_{\min }=\sum_{\ell=1}^{\left[N_{u, 1}\right]_{\leq}} N_{r_{2} \ell}$ and the maximum eigenvalue is lower limited by $\tilde{\vec{\lambda}}_{\text {max }}=\tilde{\lambda}_{\text {min }}+N_{u, 1}$.

The proof of this proposition is a direct consequence of the interlaced eigenvalue property [14, chapter 7]:

Interlaced eigenvalue property: Let $\mathbf{B}_{n}$ be a Hermitian $n \times n$ matrix with eigenvalues $\beta_{1} \leq \beta_{2} \leq \ldots \leq \beta_{n}$. Let $\mathbf{c}$ be a $n \times 1$ vector and $d$ a real-valued scalar. We can construct the bordered Hermitian matrix $\mathbf{B}_{n+1}$ of size $(n+1) \times(n+1)$ :

$$
\mathbf{B}_{n+1}=\left(\begin{array}{cc}
\mathbf{B}_{n} & \mathbf{c} \\
\mathbf{c}^{H} & d
\end{array}\right) \text {. }
$$

The eigenvalues $\lambda_{1} \leq \lambda_{2} \leq \ldots \leq \lambda_{n+1}$ of $\mathbf{B}_{n+1}$ are interlaced with those of $\mathbf{B}_{n}: \lambda_{1} \leq \beta_{1} \leq \lambda_{2} \leq \beta_{2} \leq \ldots \leq$ $\beta_{n} \leq \lambda_{n+1}$.

Proof of Proposition 3: First we show that the eigenvalues of $\mathbf{A}$ are bounded by $\lambda_{\min }$ and $\lambda_{\max }$. We define the $2 N_{u, 1} \times 2 N_{u, 1}$ real-valued symmetric Toeplitz matrix $\tilde{\mathbf{A}}_{2 N_{u, 1}}$ similarly as the matrix $\mathbf{A}$ (see (35)). The first row of $\tilde{\mathbf{A}}_{2 N_{u, 1}}$ is given by $\left[a(0), \ldots, a\left(2 N_{u, 1}-1\right)\right]$, with $a(n)=$ $\sum_{\tilde{\mathbf{A}}_{\ell=1}}^{L_{r}} N_{r, \ell} \sum_{m=0}^{M_{r, \ell}}(-1)^{m} \delta\left(n-m N_{r, \ell}\right)$. Note that the matrix $\tilde{\mathbf{A}}_{2 N_{u, 1}}$ can be decomposed as

$$
\tilde{\mathbf{A}}_{2 N_{u, 1}}=\left(\begin{array}{cc}
\mathbf{A} & \mathbf{x} \\
\mathbf{x}^{H} & \mathbf{y}
\end{array}\right)
$$

where $\mathbf{x}$ is $N_{u} \times\left(2 N_{u, 1}-N_{u}\right)$ and $\mathbf{y}$ is symmetric $\left(2 N_{u, 1}-\right.$ $\left.N_{u}\right) \times\left(2 N_{u, 1}-N_{u}\right)$. The dimension of $\tilde{\mathbf{A}}_{2 N_{u, 1}}$ is a power of 2, so the Hadamard matrix $\mathbf{H}_{2 N_{u, 1}}$ can be used to diagonalize it, resulting in the eigenvalues $\lambda_{\min }=\sum_{\ell=1}^{\left[2 N_{u, 1}\right]_{\leq}} N_{r, \ell}$ and $\lambda_{\max }=\lambda_{\min }+2 N_{u, 1}$. The interlaced eigenvalue property implies that the eigenvalues of $\mathbf{A}$ are bounded by the minimum and maximum eigenvalue of $\tilde{\mathbf{A}}_{2 N_{u, 1}}$.

Secondly, we show that the eigenvalues of $\mathbf{A}$ are bounded by $\tilde{\lambda}_{\min }$ and $\tilde{\lambda}_{\max }$. We define the $N_{u, 1} \times N_{u, 1}$ Toeplitz matrix $\tilde{\mathbf{A}}_{N_{u, 1}}$. We can write $\mathbf{A}$ as

$$
\mathbf{A}=\left(\begin{array}{cc}
\tilde{\mathbf{A}}_{N_{u, 1}} & \mathbf{x} \\
\mathbf{x}^{H} & \mathbf{y}
\end{array}\right)
$$

where $\mathbf{x}$ is $N_{u, 1} \times \bar{N}_{u, 1}$ and $\mathbf{y}$ is $\bar{N}_{u, 1} \times \bar{N}_{u, 1}$. The interlaced eigenvalue property implies that the eigenvalues of $\tilde{\mathbf{A}}_{N_{u, 1}}$ are bounded by the minimum and maximum eigenvalue of $\mathbf{A}$. The eigenvalues of $\tilde{\mathbf{A}}_{N_{u, 1}}$ can be found by multiplying it with the Hadamard matrix $\mathbf{H}_{N_{u, 1}}$, yielding the eigenvalues $\tilde{\lambda}_{\min }=$ $\sum_{\ell=1}^{\left[N_{u, 1}\right]_{\leq}} N_{r, \ell}$ and $\tilde{\lambda}_{\max }=\tilde{\lambda}_{\min }+N_{u, 1}$. Hence, it follows that $\lambda_{\text {min }}$ is an upper bound for the smallest eigenvalue of $\mathbf{A}$ and $\tilde{\lambda}_{\max }$ is a lower bound for the maximum eigenvalue of A. $\square$

Note that the redundant energy is given by $P_{r}=$ $E_{s} \sum_{m=1}^{N_{u}}\left(1-\frac{\lambda_{m}}{N}\right) /\left(\frac{\lambda_{m}}{N}\right)$, where $\lambda_{m}$ are the eigenvalues of $\mathbf{A}$. The smaller the relative difference between the eigenvalues, the smaller the redundant energy, with a minimum when all eigenvalues are equal. When $2 N_{u, 1} \leq N_{r,\left[2 N_{u, 1}\right]_{<} \text {, it }}$ follows from the previous derivations that $\lambda_{\min }=0$ and

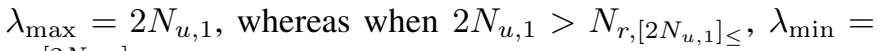
$\sum_{\ell=1}^{\left[2 N_{u, 1}\right]_{\leq}} \geq 2 N_{u, 1}$ and $\lambda_{\max } \geq 4 N_{u, 1}$. In the latter case, the relative difference between the eigenvalues is smaller, such that the redundant energy in the latter case will be relatively smaller than in the former case. Hence, this provides us a rule of thumb to select the number of redundant carriers and the length of the unique word.

\section{Numerical Results}

In this section, we analytically evaluate the data energy $P_{d}$, redundant energy $P_{r}$ and total energy $P_{t}$ as function of the system parameters, i.e. the FFT size $N$, the number of redundant carriers $N_{r}$ and the length of the unique word $N_{u}$.

In Figure 3, the energy is shown as function of the number of redundant carriers, assuming that the number of redundant carriers is the minimum possible, i.e. $N_{r}=N_{u}$, for $N=32$. As expected, the data energy $P_{d}$ equals $N_{d} E_{s}=\left(N-N_{r}\right) E_{s}$. The symmetry of the redundant energy around $N / 2=16$ can clearly be seen in the figure. Also the symmetries of the total energy in the intervals $\left[N_{r, i}^{x}, N_{r, i}^{x}+\frac{N}{2^{i+1}}\right]$ around $N_{r, i}^{x}+\frac{N}{2^{i+2}}$, where $N_{r, i}^{x}=N \sum_{\ell=1}^{i} 2^{-\ell}=N-\frac{N}{2^{i}}$, for $i=0, \ldots, \log _{2} N-$ 1 can be observed in the figure. As expected from our analysis, when $N_{r}$ is a power of 2, the redundant energy equals the data energy (the curves in the figures coincide for these values of $N_{r}$ ). Further, we observe that the redundant and total energy needed for odd values of $N_{r}$ are larger than for even values of $N_{r}$. This can be explained with the closed form expression (31) for the total energy. Consider the total energy for the values $N_{r}=X$ and $N_{r}=X+1$, where $X$ is assumed to 


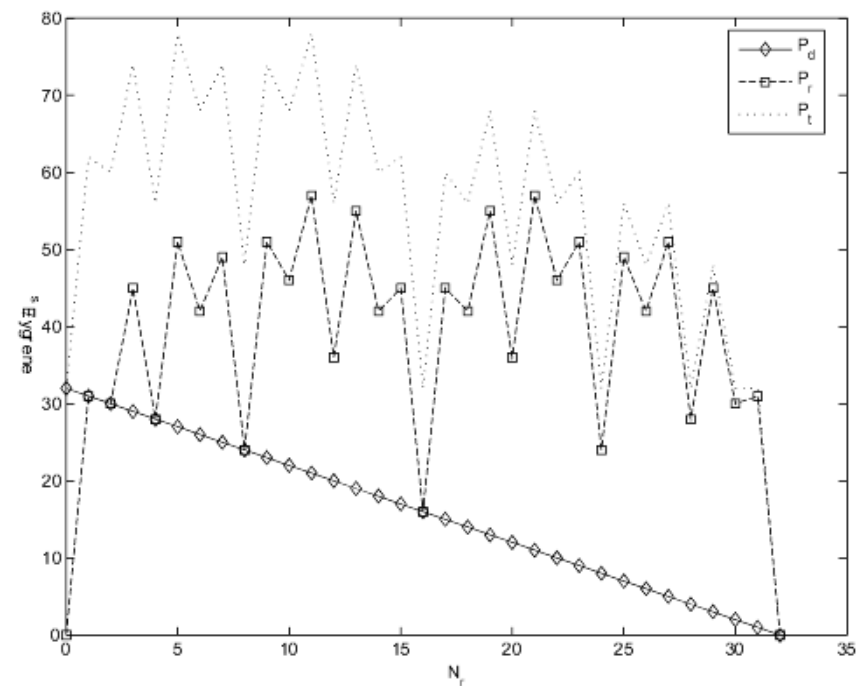

Fig. 3. Data, redundant and total energy as function of $N_{r}, N_{u}=N_{r}$, $N=32$.

be even. Hence, $X$ can be written as $X=\sum_{\ell=1}^{L_{r}} N_{r, \ell}$, where $N_{r, L_{r}}>1$. The difference between the total energies can easily be derived from (31):

$$
P_{t}(X+1)-P_{t}(X)=E_{s}(N-2)-E_{s} \sum_{\ell=1}^{L_{r}} \frac{N}{N_{r, \ell}} .
$$

As $N_{r, 1} \leq N / 2$ and $N_{r, L_{r}}>1$, the sum $\sum_{\ell=1}^{L_{r}} \frac{N}{N_{r, \ell}}$ can be upper limited by

$$
\sum_{\ell=1}^{L_{r}} \frac{N}{N_{r, \ell}} \leq N \sum_{\ell=1}^{\log _{2} N-1} \frac{1}{2^{\ell}}=N-2 .
$$

Hence, it follows that $P_{t}(X+1)-P_{t}(X) \geq 0$. Further, considering that $P_{t}(X)=E_{s}(N-X)+P_{r}(\bar{X})$, it follows that $P_{r}(X+1)-P_{r}(X) \geq E_{s}>0$.

Next, we evaluate the effect of the FFT size on the energies. In Figure 4, the energy (normalized on $N E_{s}$ ) is shown for different values of $N_{r}=N_{u}$. The normalized energies increase with $N$, and reach an asymptote for large $N$. Hence, for large $N$, the three energies $\left(P_{d}, P_{r}\right.$ and $P_{t}$ ) are essentially linear in $N$. For given $N$, doubling $N_{r}$ reduces $P_{d}, P_{r}$ and $P_{t}$. This can be explained as follows. The data energy equals $P_{d}\left(N_{r}\right)=\left(N-N_{r}\right) E_{s}$, such that $P_{d}\left(N_{r}\right)-P_{d}\left(2 N_{r}\right)=N_{r} E_{s}$. For the total energy, it follows from (31) that $P_{t}\left(N_{r}\right)-P_{t}\left(2 N_{r}\right)=2 E_{s} N_{r}$. Further, taking into account that $P_{t}(X)=P_{d}(X)+P_{r}(X)$, it follows that $P_{r}\left(N_{r}\right)-P_{r}\left(2 N_{r}\right)=N_{r} E_{s}$. Hence, doubling $N_{r}$ will reduce $P_{d}$ and $P_{r}$ with an amount $N_{r} E_{s}$ and $P_{t}$ with $2 N_{r} E_{s}$. Further, it can be observed in the figure that the normalized energies are the same when $N$ and $N_{r}=N_{u}$ are multiplied with a power of 2, i.e. $P_{x}\left(N, N_{r}\right) /\left(N E_{s}\right)=P_{x}\left(N 2^{z}, N_{r} 2^{z}\right) /\left(N 2^{z} E_{s}\right)$, where $x=d, r, t$ and $z$ is an integer. For the case of the data energy, the derivation is straightforward taking into account that $P_{d}\left(N 2^{z}, N_{r} 2^{z}\right)=2^{z}\left(N-N_{r}\right) E_{s}=2^{z} P_{d}\left(N, N_{r}\right)$. For the total energy, it can easily be derived from the expression (31), and hence, considering $P_{t}(X)=P_{d}(X)+P_{r}(X)$, it directly follows for the redundant energy.

In the previous figures, we have restricted our attention to the case where $N_{u}=N_{r}$. In Figure 5, we consider the

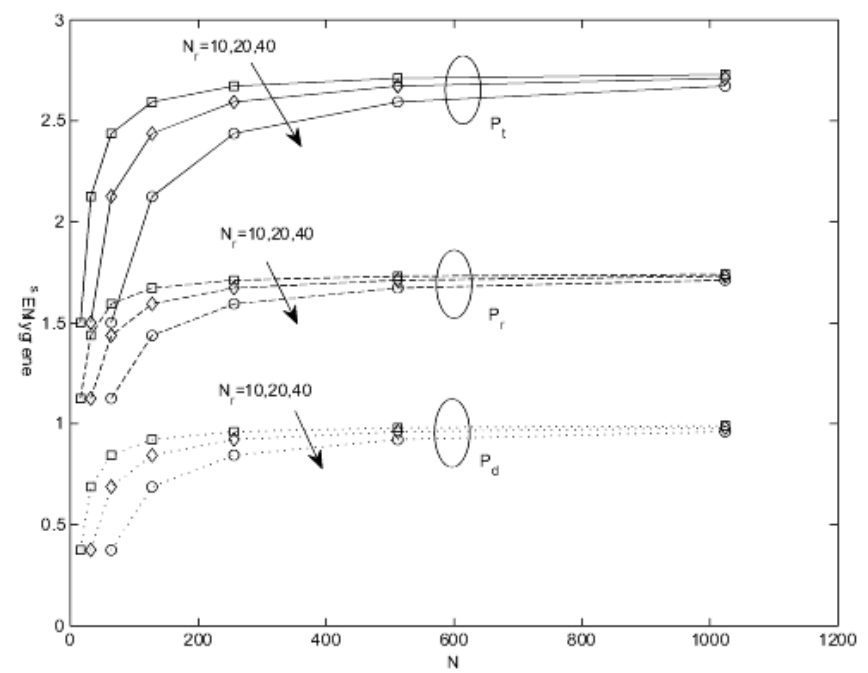

Fig. 4. Data, redundant and total energy as function of $N, N_{u}=N_{r}$.

case where the length $N_{u}$ of the unique word is varied when $N$ and $N_{r}$ are kept constant. The data energy is equal to $P_{d}=N_{d} E_{s}=\left(N-N_{r}\right) E_{s}$ and hence independent of the length of the unique word. When $N_{r}$ is a power of 2 , it was shown that the redundant energy equals $\frac{N_{d} N_{u}}{N_{r}} E_{s}$, i.e. the redundant energy linearly increases with $N_{u}$, and reaches its maximum $N_{d}$ when $N_{u}=N_{r}$. When $N_{r}$ is not a power of 2, we use the eigenvalues of the matrix $A$ to evaluate the redundant energy. Recall that the redundant energy equals $P_{r}=E_{s} \sum_{i=1}^{N_{u}} \frac{N-\lambda_{m}}{\lambda_{m}}$. It becomes minimum when all eigenvalues are equal, resulting in $P_{r, \min }=E_{s} \frac{N_{u} N_{d}}{N_{r}}$. If one or more of the eigenvalues $\lambda_{m}$ is small as compared to the other eigenvalues, the redundant energy will strongly increase. In the figure, for the cases where $N_{r}$ is not a power of 2 , we have added the curves $P_{r, \min }$ as a reference (i.e. the grey dotted lines in the figure). When $N_{r}$ is not a power of 2, the redundant energy increases faster than $P_{r, \text { min }}$. For small values of $N_{u}$, the redundant energy is essentially linear in $N_{u}$ (close to $\left.P_{r, \text { min }}\right)$, but when $N_{u}>2^{\left\lfloor\log _{2} N_{r}\right\rfloor}\left(N_{u}>8\right.$ in the figure), the redundant energy strongly increases as compared to $P_{r, \min }$. This can be explained by observing the eigenvalues of the matrix $\mathbf{A}$ for the different cases. The case $N_{r}=15$ corresponds to the case $N_{r}=2^{m}-1$ which was discussed in section IV. In this case, the eigenvalues are $\lambda=2^{m}=16$ with multiplicity $\nu=N_{u}-1$ and $\lambda=16-N_{u}$ with multiplicity 1 . The redundant energy is given by (50). For $N_{u} \ll 2^{m}$ and approximating $2^{m} / N \approx N_{r} / N$, the redundant energy (50) can be approximated by $P_{r} \approx P_{r, \text { min }}$. Hence, for small $N_{u}$, the redundant energy is approximately minimal. When $N_{u} \lesssim N_{r}$, the eigenvalue with multiplicity one, i.e. $\lambda=2^{m}-N_{u}$ becomes relatively small as compared to the other eigenvalues, which explains the strong increase of the redundant energy in the figure. In Table I, the eigenvalues and their corresponding multiplicities are shown for $N_{r}=10$. When $N_{u} \leq 8$, the relative difference between the eigenvalues is small, and hence the redundant energy is close to the minimum $P_{r, \min }$. For $N_{u}>8$, one of the eigenvalues is $\lambda=2$. As this eigenvalue is relatively small as compared to the other eigenvalues, the redundant energy is strongly increased. Note 


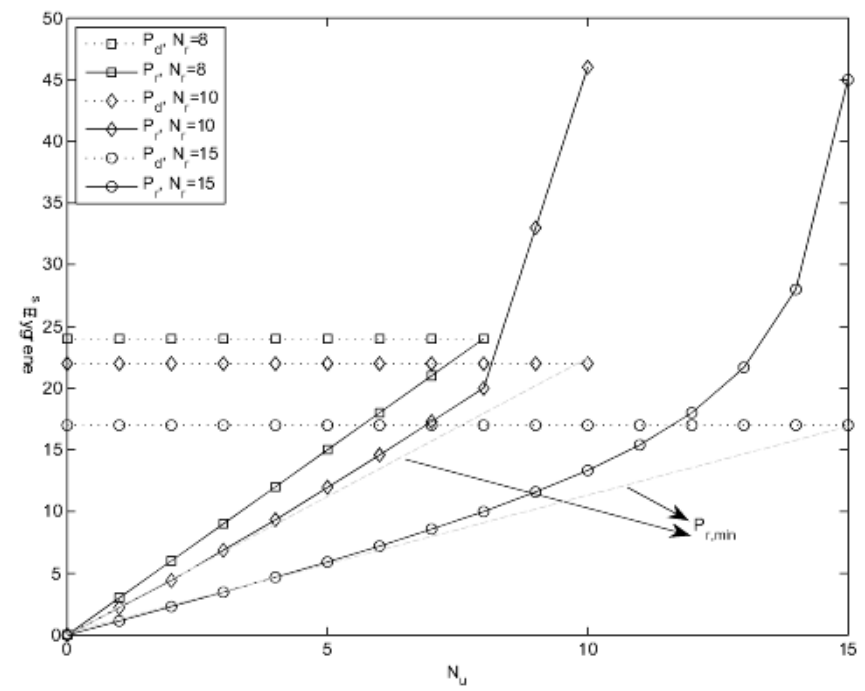

Fig. 5. Redundant energy as function of $N_{u}, N=32$.

TABLE I

EIGENVALUES $\lambda$ AND THEIR CORRESPONDING MULTIPLICITIES $\nu$ FOR $N=32$ AND $N_{r}=10$.

\begin{tabular}{|c|c|}
\hline$N_{u}$ & $\lambda(\nu)$ \\
\hline 1 & $10(1)$ \\
2 & $10(2)$ \\
3 & $8(1), 10(1), 12(1)$ \\
4 & $8(2), 12(2)$ \\
5 & $8(3), 12(1), 14(1)$ \\
6 & $8(4), 14(2)$ \\
7 & $8(5), 14(1), 16(1)$ \\
8 & $8(6), 16(2)$ \\
9 & $2(1), 8(5), 16(3)$ \\
10 & $2(2), 8(4), 16(4)$ \\
\hline
\end{tabular}

that when $N_{u}<N_{r}$, for most cases the average redundant energy is smaller than the data energy, whereas for $N_{u}=N_{r}$, the redundant energy is larger than or equal to the data energy.

\section{CONCLUSIONS}

One of the main concerns in UW-OFDM is the energy usage of the redundant carriers. In this paper, we analytically evaluate the energy necessary to transmit the redundant symbols. In [7], the authors have shown through simulations that for $N_{r}$ a power of 2, a close to uniform distribution was found to achieve the minimum average redundant energy given the restriction that the carriers in the frequency guard bands are not allowed to be used. In this paper we analytically prove that, if all carriers may be used, the uniform distribution is optimal. However, in contrast with [7], we do not restrict our attention to the case where $N_{r}$ is a power of 2. As the uniform distribution in that case is no longer optimal, we use the suboptimal split distribution from [1].

In the special case where $N_{u}=N_{r}$, i.e. when the number of redundant carriers is the minimum possible, we derive a closed form expression for the redundant and total energy, which avoids the need of extensive computations. Further, we show the existence of different symmetries in $N_{r}$ of the redundant energy and the total energy. It turns out that when $N_{r}=$ $N_{u}$, the redundant energy for odd $N_{r}$ is larger than for even $N_{r}$. Further, doubling the number of redundant carriers while keeping the number of carriers constant decreases the data, redundant and total energy proportionally with $N_{r}$, and when the number of carriers $N$ is doubled together with $N_{r}$, the energies are doubled.

In the general case where the number of redundant carriers is no longer equal to the length of the unique word, i.e. $N_{u}<N_{r}$, we analyse the redundant energy by evaluating the eigenvalues of the matrix $\mathbf{A}$. When one or more of the eigenvalues is relatively small as compared to the other eigenvalues, the redundant energy strongly increases. Hence, this situation must be avoided. In the paper we have shown that when $2 N_{u, 1} \leq N_{r,\left[2 N_{u, 1}\right]_{<}}$, the relative difference between

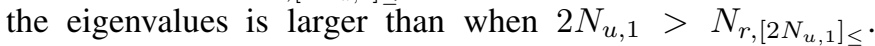
Hence, this inequality can be used as a rule of thumb in the selection of the system parameters. Further, in this paper, we have shown that in the general case, the redundant energy as function of $N_{u}$ increases faster than $P_{r, \min }$, which is the minimum possible redundant energy.

From figures 3-5 it follows that the average redundant energy is relatively large as compared to the data energy. However, this high redundant energy will not necessarily lead to a BER degradation. The explanation is as follows. If at the receiver only the data carriers were considered for data detection, the redundant carriers are thrown away and a linear data detector is used as in CP-OFDM, the high redundant energy would be a drawback, as the energy in the redundant carriers is wasted. However, if we use the information that is available in the redundant carriers to detect the data this requires more sophisticated data detectors than in CPOFDM - it is shown (see [7], [8], [10] and [15]) that for $N_{u}=N_{r}$ (which yields the maximum average redundant energy as function of $N_{u}$ ) and in the presence of a frequency selective channel, the BER for UW-OFDM is lower than that of CP-OFDM. The redundant part of the UW-OFDM system acts like an error correcting code, so the high redundant energy needed will not cause a BER degradation as long as it is compensated by the 'error correcting capability' of the UWOFDM 'code'.

\section{APPENDIX}

In this appendix, we derive the recursive expression for the total energy (30). We consider the following two cases: $2^{y}=$ $N / 2$ and $2^{y}<N / 2$.

Case 1: $2^{y}=N / 2$

In this case the total energy yields

$$
\begin{aligned}
& P_{t}\left(\frac{N}{2}+Y\right) \stackrel{(17)}{=} \quad P_{t}\left(\frac{N}{2}-Y\right)-2 Y E_{s} \\
& \stackrel{(19)}{=} P_{t}(Y)-2 Y E_{s}
\end{aligned}
$$

Case 2: $2^{y}<N / 2$

In this case the total energy can be rewritten as:

$$
\begin{aligned}
P_{t}\left(2^{y}+Y\right) \stackrel{\stackrel{17}{=}}{=} & P_{t}\left(N-2^{y}-Y\right)+\left(N-2^{y+1}-2 Y\right) E_{s} \\
= & P_{t}\left(N-2^{y+1}+2^{y}-Y\right) \\
& +\left(N-2^{y+1}-2 Y\right) E_{s} \\
\stackrel{(28)}{=} & P_{t}\left(2^{y}-Y\right)-2\left(2^{y}-Y\right)\left(\frac{N}{2^{y+1}}-1\right) E_{s}
\end{aligned}
$$




$$
\begin{array}{ll} 
& +\left(N-2^{y+1}-2 Y\right) E_{s} \\
\stackrel{(19)}{=} & P_{t}(Y)+2\left(2^{y}-2 Y\right)\left(\frac{N}{2^{y+1}-1}\right) E_{s} \\
& -2\left(2^{y}-Y\right)\left(\frac{N}{2^{y+1}}-1\right) E_{s} \\
& +\left(N-2^{y+1}-2 Y\right) E_{s} \\
= & P_{t}(Y)+\left(N-2^{y+1}-\frac{N}{2^{y}} Y\right) E_{s}
\end{array}
$$

Note that for $2^{y}=N / 2$, the expression (58) reduces to (57). Hence, combining the results yields the recursive expression (58) for all values of $0 \leq y \leq \log _{2} N-1$.

\section{REFERENCES}

[1] H. Steendam, "On the redundant carrier distribution for UW-OFDM," in Proc. 2012 Wireless Commun. Netw. Conf.

[2] J. A. C. Bingham, "Multicarrier modulation for data transmission, an idea whose time has come," IEEE Commun. Mag., vol. 31, pp. 5-14, May 1990.

[3] Transmission and Multiplexing (TM); Access Transmission Systems on Metallic Access Cables; Very High Speed Digital Subscriber Line (VDSL); Part 2: Transceiver Specification, ETSI TS 101 270-2, European Telecommunications Standards Institute, 2001.

[4] R. van Nee, G. Awater, M. Morikura, H. Takanashi, M. Webster, and K. W. Halford, "New high-rate wireless LAN standards," IEEE Commun. Mag., vol. 37, pp. 82-88, Dec 1999.

[5] B. Muquet, Z. Wang, et al., "Cyclic prefixing or zero padding for wireless multicarrier transmissions?" IEEE Trans. Commun., vol. 50, pp. 2136-2148, Dec. 2002.

[6] H. Steendam and M. Moeneclaey, "Different guard interval techniques for OFDM: performance comparison," in Proc. 2007 Multicarrier Spread-Spectrum Symp., pp. 11-24.

[7] M. Huemer, C. Hofbauer, and J. B. Huber, "The potential of unique words in OFDM," in Proc. 2010 Int. OFDM Work., pp. 140-144.

[8] A. Onic and M. Huemer, "Direct vs. two-step approach for unique word generation in UW-OFDM," in Proc. 2010 Int. OFDM Work., pp. 145149.

[9] I. S. Reed and G. Solomon, "Polynomial codes over certain finite fields," J. SIAM, vol. 8, no. 2, pp. 300-304, 1960.

[10] C. Hofbauer, M. Huemer, and J. B. Huber, "Coded OFDM by unique word prefix," in Proc. 2010 Int. Conf. Commun. Syst.

[11] D. Van Welden, H. Steendam, and M. Moeneclaey, "Frequency-domain data aided channel estimation for KSP-OFDM," in Proc. 2008 Int. Symp. Spread Spectrum Tech. Applic., pp. 613-617.

[12] D. Van Welden, H. Steendam, and M. Moeneclaey, "Time delay estimation for KSP-OFDM systems in multipath fading channels," in Proc. 2009 Int. Symp. Personal, Indoor Mobile Radio Commun.

[13] D. Van Welden, H. Steendam, and M. Moeneclaey, "Frequency offset estimation for KSP-OFDM systems channels," in Proc. 2011 Int. Work. Signal Process. Adv. Wireless Commun.

[14] C. D. Meyer, Matrix Analysis and Applied Linear Algebra. SIAM, 2000.

[15] M. Huemer, A. Onic, and C. Hofbauer, "Classical and Bayesian linear data estimators for unique word OFDM," IEEE Trans. Signal Process., vol. 59, pp. 6073-6085, Dec. 2011.

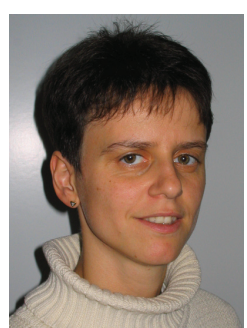

Heidi Steendam (M'01, SM'06) received the M.Sc. degree in Electrical Engineering and the Ph.D. degree in Applied Sciences from Ghent University, Gent, Belgium in 1995 and 2000, respectively. Since September 1995, she has been with the Digital Communications (DIGCOM) Research Group, Department of Telecommunications and Information Processing (TELIN), Faculty of Engineering, Ghent University, Belgium, first in the framework of various research projects, and since October 2002 as a full time Professor in the area of Digital Communi-

cations.

Her main research interests are in statistical communication theory, carrier and symbol synchronization, bandwidth-efficient modulation and coding, spread-spectrum (multi-carrier spread-spectrum), satellite and mobile communication, cognitive radio and cooperative networks. She is the author of more than 100 scientific papers in international journals and conference proceedings.

Since 2002, she is an executive Committee Member of the IEEE Communications and Vehicular Technology Society Joint Chapter, Benelux Section. She has been active in various international conferences as Technical Program Committee chair or member and Session chair. In 2004, she was the conference chair of the IEEE Symposium on Communications and Vehicular Technology in the Benelux. 